\title{
Differential genome evolution and speciation of Coix lacryma-jobi L. and Coix aquatica Roxb. hybrid guangxi revealed by repetitive sequence analysis and fine karyotyping
}

Zexi Cai ${ }^{1+}$, Huijun Liu ${ }^{1 \dagger}$, Qunyan He ${ }^{1,2}$, Mingwei Pu', Jian Chen ${ }^{3}$, Jinsheng Lai ${ }^{3}$, Xuexian $\mathrm{Li}^{4}$ and Weiwei Jin ${ }^{1 *}$

\begin{abstract}
Background: Coix, Sorghum and Zea are closely related plant genera in the subtribe Maydeae. Coix comprises 9-11 species with different ploidy levels $(2 \mathrm{n}=10,20,30$, and 40$)$. The exclusively cultivated C. lacryma-jobi $\mathrm{L}$. $(2 \mathrm{n}=20)$ is widely used in East and Southeast Asia for food and medicinal applications. Three fertile cytotypes $(2 n=10,20$, and 40$)$ have been reported for C. aquatica Roxb. One sterile cytotype $(2 n=30)$ closely related to $C$. aquatica has been recently found in Guangxi of China. This putative hybrid has been named C. aquatica HG (Hybrid Guangxi). The genome composition and the evolutionary history of C. lacryma-jobi and C. aquatica HG are largely unclear.

Results: About $76 \%$ of the genome of C. lacryma-jobi and $73 \%$ of the genome of C. aquatica HG are repetitive DNA sequences as shown by low coverage genome sequencing followed by similarity-based cluster analysis. In addition, long terminal repeat (LTR) retrotransposable elements are dominant repetitive sequences in these two genomes, and the proportions of many repetitive sequences in whole genome varied greatly between the two species, indicating evolutionary divergence of them. We also found that a novel $102 \mathrm{bp}$ variant of centromeric satellite repeat CentX and two other satellites only appeared in C. aquatica HG. The results from FISH analysis with repeat probe cocktails and the data from chromosomes pairing in meiosis metaphase showed that C. lacryma-jobi is likely a diploidized paleotetraploid species and C. aquatica HG is possibly a recently formed hybrid. Furthermore, C. lacryma-jobi and C. aquatica HG shared more co-existing repeat families and higher sequence similarity with Sorghum than with Zea.
\end{abstract}

Conclusions: The composition and abundance of repetitive sequences are divergent between the genomes of $C$. lacryma-jobi and C. aquatica HG. The results from fine karyotyping analysis and chromosome pairing suggested diploidization of $\mathrm{C}$. lacryma-jobi during evolution and C. aquatica HG is a recently formed hybrid. The genome-wide comparison of repetitive sequences indicated that the repeats in Coix were more similar to those in Sorghum than to those in Zea, which is consistent with the phylogenetic relationship reported by previous work.

Keywords: Coix, Next-generation sequencing, Repeat element, Genome structure, Karyotyping, Polyploidy, Evolution

\footnotetext{
* Correspondence: weiweijin@cau.edu.cn

${ }^{\dagger}$ Equal contributors

${ }^{1}$ National Maize Improvement Center of China, Beijing Key Laboratory of Crop Genetic Improvement, Coordinated Research Center for Crop Biology, China Agricultural University, Beijing 100193, China

Full list of author information is available at the end of the article
} 


\section{Background}

Coix lacryma-jobi L. $(2 \mathrm{n}=20)$, also commonly called adlay (Job's tears), is widely cultivated as a food and medicine plant in East and Southeast Asian countries [1]. Its grain has the highest protein content among cereal crops [2], and its seed extracts are used to treat several diseases $[3,4]$. Previous research on Coix has focused mainly on its medicinal components and their efficacy [5,6]. Genetic and genomic studies on Coix are sparse, although there are some reports on cytogenetic analysis [7-9], genetic linkage map development [10], construction of BAC libraries [11], and isolation of prolamin gene families [12,13].

The genus Coix belongs to Maydeae of Poaceae family and is the closest group to the genera Zea, Tripsacum, and Sorghum [14]. The places of the origin of the three genera are very different. Zea originates from America, and Sorghum originates from Africa, while the genus Coix is indigenous to Southeast Asia [1]. Previous studies have shown that Coix comprises of 9-11 species with different levels of ploidy. Tetraploids $(2 \mathrm{n}=20)$ predominate, including the exclusively cultivated Coix lacryma-jobi L., while diploids $(2 \mathrm{n}=10)$, hexaploids $(2 \mathrm{n}=30)$, and octoploids $(2 n=40)$ with a basic chromosome number of $x=5$ are less frequent [15]. Coix aquatica Roxb. has been reported to have three fertile cytotypes $(2 n=10,20$ and 40$)$. A sterile cytotype $(2 n=30)$ of very close botanical relationship to C. aquatica was found in southwest China [16]. The results from genomic in situ hybridization (GISH) demonstrated that 20 out of its 30 chromosomes are highly homologous to the chromosomes of C. lacryma-jobi [7]. The genomic and evolutionary information of C. lacrymajobi and this sterile $C$. aquatica remains elusive. Furthermore, the phylogenetic relationship between Coix and Zea/Sorghum is still unclear. Comparative analysis of genomic DNA sequences may contribute to answer those questions.

The majority of the genomic sequences in higher eukaryotes are repetitive elements including transposable elements and satellite repeats [17]. Large-scale accumulation of satellites and transposable elements serves as a major driving force for genome expansion [18,19]. Most long terminal repeat (LTR) retrotransposable elements disperse throughout plant chromosomes, while some elements locate in specific regions of chromosomes [20]. Satellite DNA sequences, consisting of short tandem repeats, share a common structural feature [21]. They are usually located in specific chromosomal regions and may be broadly classified as centromeric, subtelomeric, or intercalary repetitive sequences [22]. Satellite repeats are very powerful tools for chromosome identification, chromosome karyotyping, and comparative genome analysis [23,24]. Because of similar chromosome lengths and/or arm ratios and lack of sufficient landmarks, adequate identification of Coix chromosome pairs remains challenging. Therefore, identification and characterization of major repetitive sequences are essential for understanding the organization and structure of Coix genomes.

In this study, we sequenced $\sim 6 \mathrm{~Gb}$ genomic sequences of C. lacryma-jobi and $12 \mathrm{~Gb}$ genomic sequences of $C$. aquatica HG, and found highly dynamic nature of these two genomes during evolution. Our karyotyping analysis revealed that C. lacryma-jobi is a diploidized paleotetraploid species, and C. aquatica HG is recently formed hybrid between $C$. lacryma-jobi and a distantly related species. Comparative repeat sequence analysis supported that Coix is closer to Sorghum than to Zea. The findings in this study advance our understanding of Coix genome evolution and shed new light on the genomics of the Poaceae family.

\section{Results}

Low-coverage sequencing of C. lacryma-jobi and

C. aquatica HG genomes and repetitive sequence analysis

To better understand the Coix genus at the genome level, we determined the genome size of the two Coix species using flow cytometry with the maize inbred line B73 (approximately 2,300 $\mathrm{Mb}$ ) as a reference genome (Additional file 1: Figure S1). The genome size of $C$. lacryma-jobi was approximately 1,684 Mb (Table 1), similar to the previous report [25]. C. aquatica HG had a genome of approximately 2,335 $\mathrm{Mb}$ (Table 1), about 1.4-fold of the C. lacryma-jobi genome.

We sequenced (Table 1) and used cluster-based repeat identification and classification method [26] to analyze the data. Except Penelope retrotransposon and P transposon superfamilies [27], all other plant transposable elements and repetitive DNA were detected in these two species, including LTR and LINE/SINE retrotransposons, Mutator and En-Spm transposons, satellites, and rDNA. The proportion of total repetitive sequences in whole genome is $75.54 \%$ in C. lacryma-jobi and $72.88 \%$ in C. aquatica HG. The proportion of different types of repetitive DNA was displayed in Table 2.

We found that retroelements occupied $69.08 \%$ of the C. lacryma-jobi genome and $63.83 \%$ of the C. aquatica HG genome (Table 2). The two dominant families of retroelements are Ty1/Copia and Ty3/Gypsy retrotransposons. The Ty1/Copia occupied $39.57 \%$ of the C. lacryma-jobi genome and $31.12 \%$ of the C. aquatica HG genome, while the Ty3/Gypsy occupied $29.11 \%$ and $32.56 \%$, respectively. The most abundant type I transposon was the Spm class of transposons, and the Mutator was also enriched in Coix.

We believed that the abundance of many repeats varied greatly between these two species. To test this hypothesis, we conducted a comparative analysis by combining reads of these two species and performing cluster-based repeat 
Table 1 Size estimation and sequencing of C. lacryma-jobi and C. aquatica HG genomes

\begin{tabular}{lccccc}
\hline Species & Chromosome number (2n) & Genome size (Gb) & Number of reads & Total read length (Mb) & Genome coverage \\
\hline C. lacryma-jobi & 20 & 1.684 & $61,869,688$ & 6186.97 & $1.84 \times$ \\
C. aquatica & 30 & 2.335 & $128,853,294$ & 12885.33 & $2.76 \times$ \\
\hline
\end{tabular}

identification and classification on the combined reads $[28,29]$. In spite of lacking statistical examination on these data, we found that CL1, a Ty1/Copia member, occupied $3.44 \%$ of the C. lacryma-jobi genome but only $1.16 \%$ of the $C$. aquatica HG genome, and other Ty1/Copia elements including CL4, CL8, CL9, CL11, and CL27 all displayed a similar pattern (Supplemental Data 2). Together, Ty1/Copia retroelements have a higher percentage in $C$. lacryma-jobi than in C. aquatica HG. Similarly, some clusters of Ty3/Gypsy also showed genome proportion change, for example, CL2 and CL414 occupies $1.7 \%$ and $0.000134 \%$ of the C. lacryma-jobi genome respectively, while $1.07 \%$ and $0.00707 \%$ of the C. aquatica HG genome respectively. To further verify these results, we conducted FISH on the chromosomes of the two species using CL2 and CL414 as probes (Additional file 1: Figure S2). CL2 probe exhibited intense signal in both species. In C. aquatica HG, 20 out of 30 chromosomes showed stronger signals than the other 10 chromosomes. CL414 probe only showed faint signal in C. lacryma-jobi, while intense signal

Table 2 Repetitive sequences and their proportions in C. Iacryma-jobi and C. aquatica HG

\begin{tabular}{lcr}
\hline \multicolumn{3}{c}{ Proportion in two genomes (\%) } \\
\hline Repeat family & C. lacryma-jobi & C. aquatica \\
Retrotransposon & 69.08 & 63.83 \\
Ty1/Copia & 39.57 & 31.12 \\
Ty3/Gypsy & 29.11 & 32.56 \\
Unclassified LTR & 0.28 & 0.12 \\
LINE/SINE & 0.12 & 0.03 \\
Transposon & 4.38 & 3.30 \\
hAT & 0.03 & 0.06 \\
Mutator & 0.57 & 0.37 \\
RC/Helitron & 0.02 & 0.04 \\
En-Spm & 3.64 & 2.42 \\
PIF-Harbinger & 0.09 & 0.24 \\
TC1-Mariner & 0.01 & 0.14 \\
Other & 0.02 & 0.03 \\
rDNA & 0.43 & 0.56 \\
SnRNA & $\mathrm{ND}$ & $\mathrm{ND}$ \\
Satellite & 0.60 & 4.89 \\
Unclassified & 1.05 & 0.30 \\
Total & 75.54 & 72.88 \\
\hline Note: & &
\end{tabular}

Note: snRNA, small noncoding RNA; ND, not detected. was observed on C. aquatica HG chromosomes. Similar to the signal of CL2 probe, 20 out of the 30 chromosomes in C. aquatica HG showed stronger signal of CL414 probe than the other 10 chromosomes. The distribution and proportion of Ty1/Copia and Ty3/Gypsy family members were illustrated in Figure 1. Our sequencing results revealed that four families (CL286, CL411, CL429, and CL472) were present only in one species but absent in the other species. We further confirmed the results of CL411 and CL429 by FISH (Additional file 1: Figure S3). This varied abundance of the LTR retroelements in the two species may result from sweep or accumulation of the same families, or the rapid change during polyploidization [30].

Our results showed that the satellite DNA made up only $0.60 \%$ of the C. lacryma-jobi genome in contrast to $4.89 \%$ of the C. aquatica HG genome. We also found that the most abundant repeats in these two species were completely different, and the proportion of the coexisting satellite repeats varied greatly in these two genomes (Table 3), indicating rapid evolution of satellite repeats in Coix. In particular, two distinct satellite repeats (SatS1 and SatS2) made up approximately $2.17 \%$ of the $C$. aquatica HG genome (Table 3 ). The rDNA proportion was similar in the two genomes. The information of other repeat families was presented in the Additional file 2.

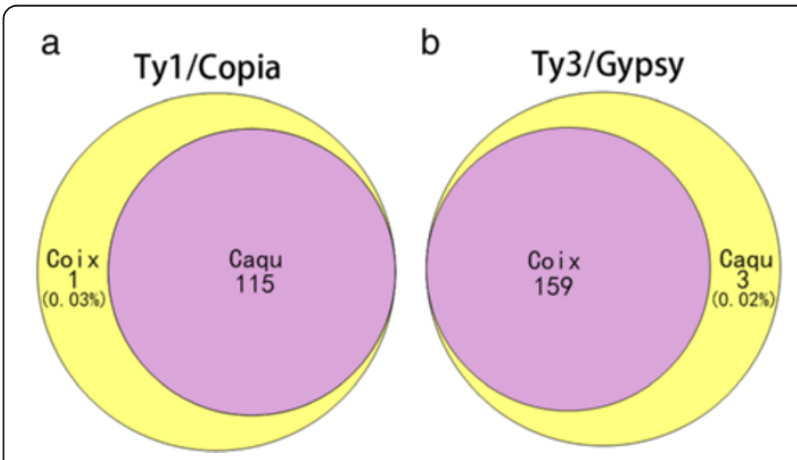

Figure 1 Ty1/Copia and Ty3/Gypsy cluster distribution in C. lacryma-jobi and C. aquatica HG. Ty1/Copia and Ty3/Gypsy cluster distribution in C. lacryma-jobi and C. aquatica HG. (a) C. lacryma-jobi and C. aquatica HG had 115 Ty1/Copia clusters in common, and only one distinct cluster was detected only in C. lacryma-jobi. (b) C. lacryma-jobi and C. aquatica HG shared 159 common Ty3/ Gypsy clusters, and 3 clusters were detected only in C. aquatica HG. The percentage represents the proportion of corresponding clusters in the genome. 
Table 3 The proportion, length, and location of satellite repeats in C. lacryma-jobi and C. aquatica HG

\begin{tabular}{lcccccccl}
\hline Satellite & \multicolumn{2}{c}{ C. lacryma-jobi } & \multicolumn{2}{c}{ C. aquatica } & Length (bp) & Location \\
\cline { 2 - 5 } & Rank & GP (\%) & & Rank & GP (\%) & & \\
\hline SatS1 & - & - & 1 & 1.34 & 358 & Subtelomere \\
SatS2 & - & - & 2 & 0.83 & 369 & Subtelomere \\
45S rDNA & 1 & 0.32 & 6 & 0.55 & - & & Chr 1S \\
SatS3 & 2 & 0.29 & 5 & 0.57 & 187 & Subtelomere \\
CentX* & 3 & 0.16 & 4 & 0.67 & 153 & Centromere \\
5S rDNA & 4 & 0.11 & 9 & 0.05 & - & Chr 4 L \\
SatS4 & 5 & 0.07 & 7 & 0.46 & 184 & Subtelomere \\
SatS5 & 6 & 0.06 & 3 & 0.79 & 185 & Subtelomere \\
Telomere & 7 & 0.02 & 8 & 0.23 & 7 & Telomere \\
\hline
\end{tabular}

${ }^{*}$ CentX is the 153 bp centromere satellite [7]; GP, genome proportion.

\section{A novel centromeric satellite repeat was identified in C. aquatica $\mathrm{HG}$}

It has been demonstrated that ten chromosomes in $C$. aquatica HG with less homology to C. lacryma-jobi harbor low copy number of centromeric repeat CentX and a large amount of centromeric retrotransposon CRC [7]. To identify possible new centromeric repeats in these ten chromosomes, we used an OsCenH3 antibody specific for the 30 centromeres in C. aquatica HG to perform ChIPseq assay (Additional file 1: Figure S4). Quantitative ChIP PCR showed significant relative enrichment of CentX and CRC in the ChIPed DNA (Figure 2), suggesting that CentX and CRC might be associated with CenH3, the functional centromere marker. In the absence of a reference genome, we mapped ChIP-seq data to the clusters of the C. aquatica HG genome for cluster-based repeat identification. The result also showed the significant relative enrichment of CentX and CRC. Besides, we identified a novel CentX variant in $C$. aquatica $\mathrm{HG}$. In contrast to

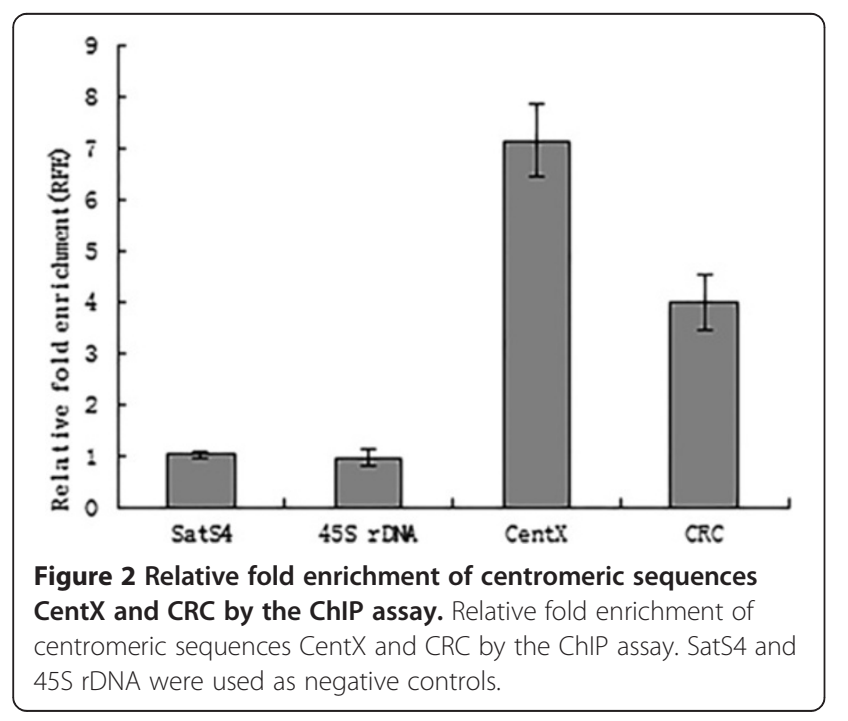

the typical size of $153 \mathrm{bp}$ for CentX, the monomer of this CentX variant had $102 \mathrm{bp}$ and accounted for $1.21 \%$ of the total CentX in the ChIPed DNA. According to our whole genome sequencing data, this variant made up $0.45 \%$ of the total CentX and $0.003 \%$ of the C. aquatica HG genome. To test whether this variant is specifically located on the 10 chromosomes less homologous to the C. lacrymajobi chromosomes, we conducted FISH using the sequence of the $102 \mathrm{bp}$ CentX variant and the sequence of the $51 \mathrm{bp}$ missing from the CentX variant but in the typical CentX as separate probes (Figure 3). Both of the $102 \mathrm{bp}$ variant and the $51 \mathrm{bp}$ fragment did not show a different distribution pattern compared with the typical CentX. The extremely faint signals from the two probes showed a similar intensity on the 10 chromosomes less homologous to C. lacryma-jobi chromosomes. These results indicate that the 102 bp variant may not be specific to the 10 chromosomes.

\section{The karyotype of C. lacryma-jobi and C. aquatica HG}

We performed karyotyping analysis on C. lacryma-jobi and $C$. aquatica $\mathrm{HG}$ using the satellite repeats, $5 \mathrm{~S}$ rDNA and $45 \mathrm{~S} \mathrm{rDNA}$ as probes (Figure 4). The signal of the centromeric satellite repeat CentX was large on the Chromosomes pair 2, strong on the chromosomes pairs 4, 5, 8 and 9, weak on the chromosomes pairs 1, 3, 7 and 10, and faint on the chromosomes pair 6 . The $45 \mathrm{~S}$ rDNA probe appeared as one large signal at the end of the short arm of the chromosome pair 1. The signal from the $5 \mathrm{~S}$ rDNA was located at the peri-centromeric region of the long arm of the chromosome pair 4. It is generally agreed that, SatS3, SatS5, and SatS4 signals sequentially approach the telomere at the subtelomeric region. Thus, using these five repeat probes, we expected to identify the individual somatic chromosome in $C$. lacryma-jobi. The signal patterns on C. lacryma-jobi chromosomes were shown in Figure 4e. We prepared an ideogram displaying the position and fluorescence intensity of satellite repeats on C. lacryma-jobi chromosomes (Figure 4f). The relative length of each chromosome pair was listed in Additional file 1: Table S1.

We used a similar approach to determine the karyotype of $C$. aquatica HG (Figure 5). By using the CentX and $C$. lacryma-jobi genomic DNA probes, we found that 20 out of the 30 chromosomes of $C$. aquatica HG were highly homologous to the chromosomes of C. lacryma-jobi. We then further karyotyped these 20 chromosomes by sequential FISH using the following three probe cocktails: 1) $45 \mathrm{~S}$ rDNA and 5S rDNA; 2) co-existing satellites SatS3, SatS4 and SatS5; 3) SatS1 and SatS2. Signals from the coexisting satellite probe cocktail showed not only different distribution patterns in C. aquatica HG compared with C. lacryma-jobi, but also a pronounced asymmetric distribution on each chromosome pair (SatS3, SatS4 and SatS5 


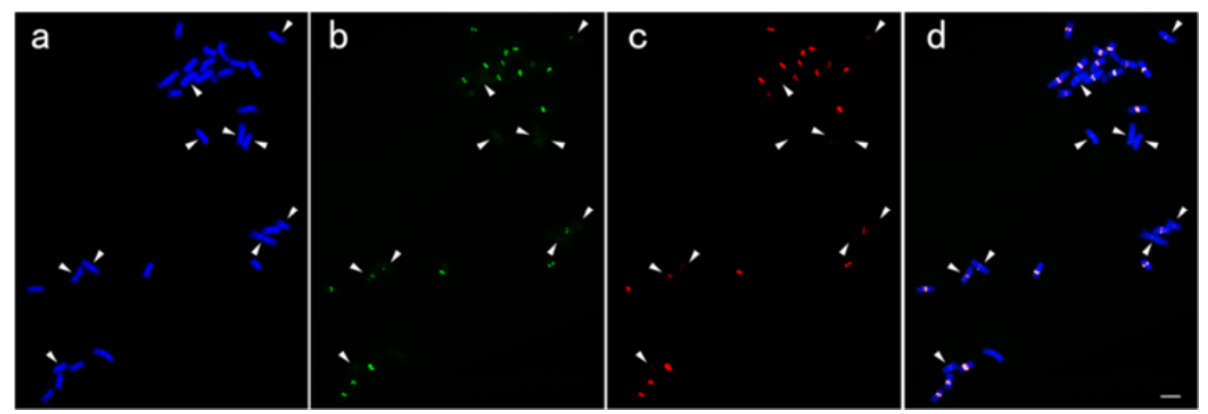

Figure 3 FISH with the 102 bp and 51 bp fragments of CentX oligonucleotide as probes. FISH with the 102 bp and 51 bp fragments of CentX oligonucleotide as probes. (a) The mitotic metaphase chromosomes of $\mathrm{C}$. aquatica HG. The arrowhead pointed the 10 chromosomes less homologues to C. lacryma-jobi chromosomes; (b) The FISH signals from the 102 bp CentX fragment (green); (c) The FISH signals from the 51 bp CentX fragment (red); (d) The merged imagine, bar $=5 \mu \mathrm{m}$.

signals were on Chr. 1, 1' and 4, 4'). We confirmed this observation by FISH on pachytene chromosomes (Figure 6). Under the condition of enhanced visualization of chromosome pairing, SatS3 and SatS5 signals on the homologous chromosome pairs appeared as a pattern of contrasting presence on one chromosome and absence on the paired one, or large amount on one chromosome and small amount on the paired one (Figure 6).

Next, we numbered the remaining 10 chromosomes as A1 to A10 and stained them with the probe cocktails. The signal distribution patterns of the probes on A1-A10 chromosomes were quite different from the patterns on the other 20 chromosomes. A faint 5S rDNA signal appeared near the end of the long arm of Chr. A7. SatS5 was absent on chromosomes A5 and A6, and 45S rDNA signal was absent on all the 10 chromosomes, indicating a different origin of this set of chromosomes compared with the other 20 chromosomes. We made a putative ideograph displaying the position and fluorescence intensity of satellites on C. aquatica HG chromosomes (Figure 7). The relative length of chromosome pairs was listed in Additional file 1: Table S2.

\section{Determination of the ploidy level of C. lacryma-jobi and C. aquatica HG}

Because certain Coix species have ten chromosomes [31], it is possible that C. lacryma-jobi is a tetraploid and $C$. aquatica HG is a hexaploid. According to previous reports, $C$. aquatica should be fertile and able to propagate by fertilization [32]. However, our karyotyping analysis revealed 10 chromosome pairs in C. lacrymajobi and 10 paired plus 10 unpaired chromosomes in C. aquatica HG, implying the absence of any typical characteristics of a tetraploid or hexaploid genome (Figures 4 and 5). We then determined chromosome pairing during metaphase $\mathrm{I}$ in $C$. lacryma-jobi and $C$. aquatica $\mathrm{HG}$ using CentX and CRC probes. Consistent with the previous report [8], our cytogenetic analysis showed that all the examined C. lacryma-jobi meiotic cells had 10 bivalents, while except one cell with an ambiguous trivalent, the rest of the examined $C$. aquatica HG meiotic cells had 10 bivalents and 10 univalents (Table 4). We also analyzed pollen viability of the C. aquatica HG using $\mathrm{I}_{2}$-KI dying method, and found that the pollen grains were sterile (Additional file 1: Table S3). Thus, based on the fact that $C$. aquatica HG is reproduced by vegetative propagation [8] and our findings, we believed that the C. aquatica HG might be recently formed hybrid and C. lacryma-jobi is a diploidized paleotetraploid species.

\section{Comparative analysis on the repeat sequence in Coix, Zea and Sorghum}

We performed classification analysis of LTR for the $C$. lacryma-jobi and C. aquatica HG using plant Ty1/Copia clades including Tos17, SIRE1/Maximus, Tnt1, Angela, Tont1, Reina and Bianca [33]. As illustrated in Figure 8a, Coix Ty1/Copia elements were distributed in six clades except the Tos17 clade. The most abundant retroelements in Coix is the SIRE1/Maximus family members, which is also abundant in maize genome [34]. Our results show that all abundant Ty1/Copia families in Sorghum and Zea were also present in Coix, indicating a close relationship of these three genera.

Additionally, we analyzed seven well characterized Ty3/Gypsy clades including CRM, Hopscotch, Tekay, Galadriel, Athila, Ogre and Tat [33]. We found Tekay, CRM, Reina, Athila, and Tat in Coix (Figure 8b). Huck in Tekay has been shown to be one of the four most dominant retroelements in Zea mays, and a moderaterepetitive DNA in Sorghum bicolor [35]. Leviathan in Tat was found to be abundant in Sorghum bicolor, and moderately abundant in Zea mays with no recent activities [35]. We found that Huck shared one node with coix_CL100 (0.238\%) and caqu_CL82 (0.361\%), and Leviathan also shared one node with coix_CL18 (0.864\%) 

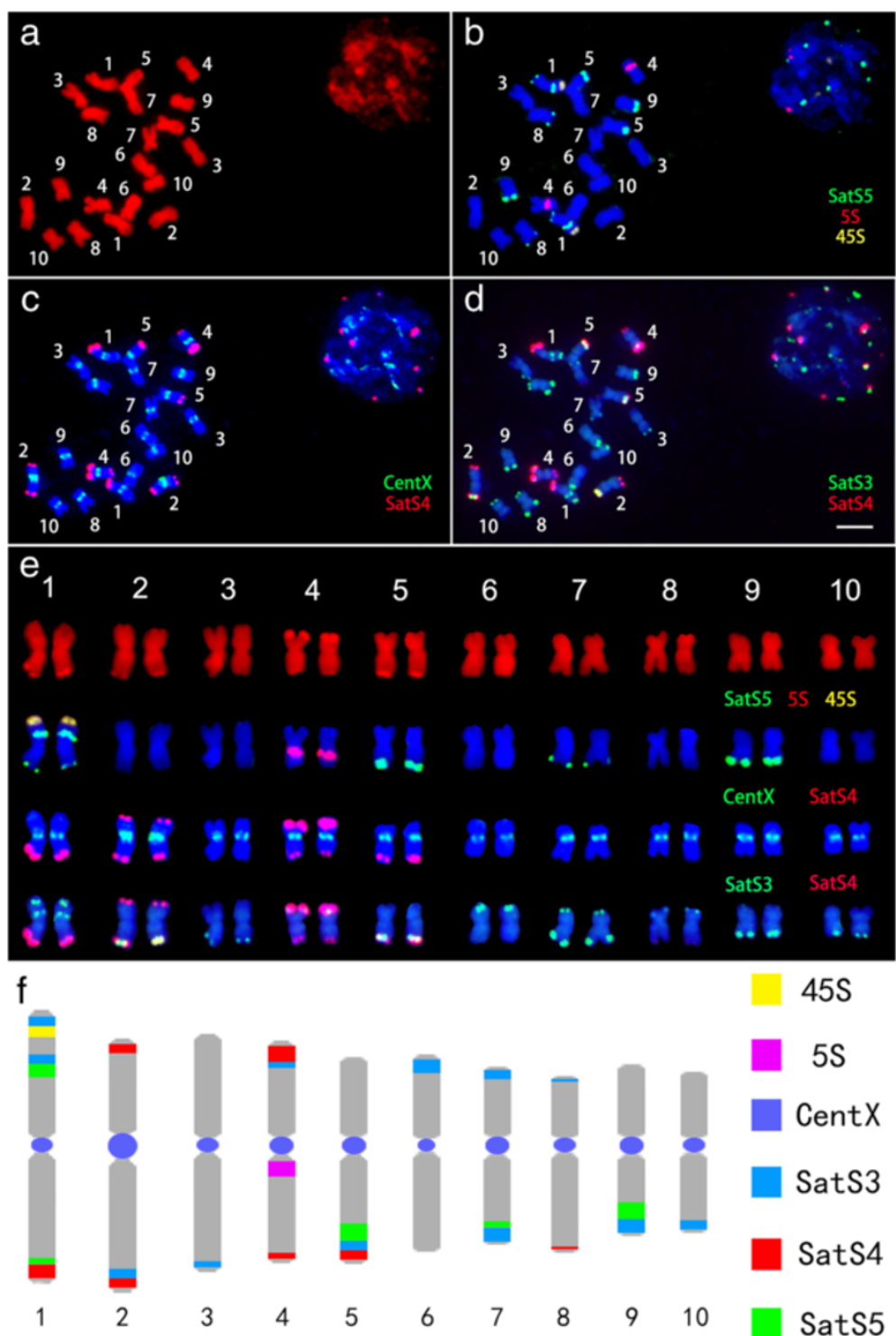

$45 S$

Figure 4 The karyotype and ideograph for C. lacryma-jobi mitotic metaphase chromosomes. The karyotype and ideograph for C. lacryma-jobi mitotic metaphase chromosomes. (a) Mitotic metaphase chromosomes were counterstained with DAPI and pseudocolored in red and chromosomes are numbered according to our karyotyping analysis; (b) FISH with the probe cocktail of SatS5 (green), 5S (red) and 45S (yellow); (c) The same spread was reprobed with the probe cocktail of CentX (green) and SatS4 (red); (d) The same spread was reprobed with the probe cocktail of SatS3 (green) and SatS4 (red); (e) Individual chromosomes displayed according to the results in (a-d); (f) The Ideograph showing the position and intensity of CentX(blue), SatS3 (light-blue), SatS4 (red), SatS5 (green), $45 S$ rDNA (yellow) and $5 S$ rDNA (purple), bar $=5 \mu \mathrm{m}$.

and caqu_CL8 (1.11\%), indicating that the divergence of Coix and Sorghum might be later than that of Sorghum and Zea.

We downloaded paired-end sequences of Zea mays (the inbred line B73), Zea luxurians, and Sorghum bicolor (the inbred line Tx378) from http://sra.dnanexus. $\mathrm{com} /$, and combined all the sequences. We found that repetitive sequences account for $79 \%$ of the maize genome and $59.4 \%$ of the sorghum genome which is very similar to the previous reports $[18,19]$. Distribution of major repeat families in the five species showed that the species-specific clusters occupied $2.18 \%$ of the C. aquatica HG genome and $8.62 \%$ of the sorghum genome, but were not detected in the other three species (Figure 9a). The co-existing clusters made up $22.98 \%$ of the C. aquatica HG genome, 26.16\% of the Coix lacryma-jobi genome, 19.33\% of the Zea mays genome, 21.22\% in the Zea luxurians genome, and $13.58 \%$ of the Sorghum bicolor genome. Coix-Sorghum co-existing clusters accounted for $12.42 \%$ of the C. aquatica HG genome, $19.16 \%$ of the Coix lacrymajobi genome, and $21.34 \%$ of the Sorghum bicolor genome. Only a few co-existing clusters were found in Zea and 

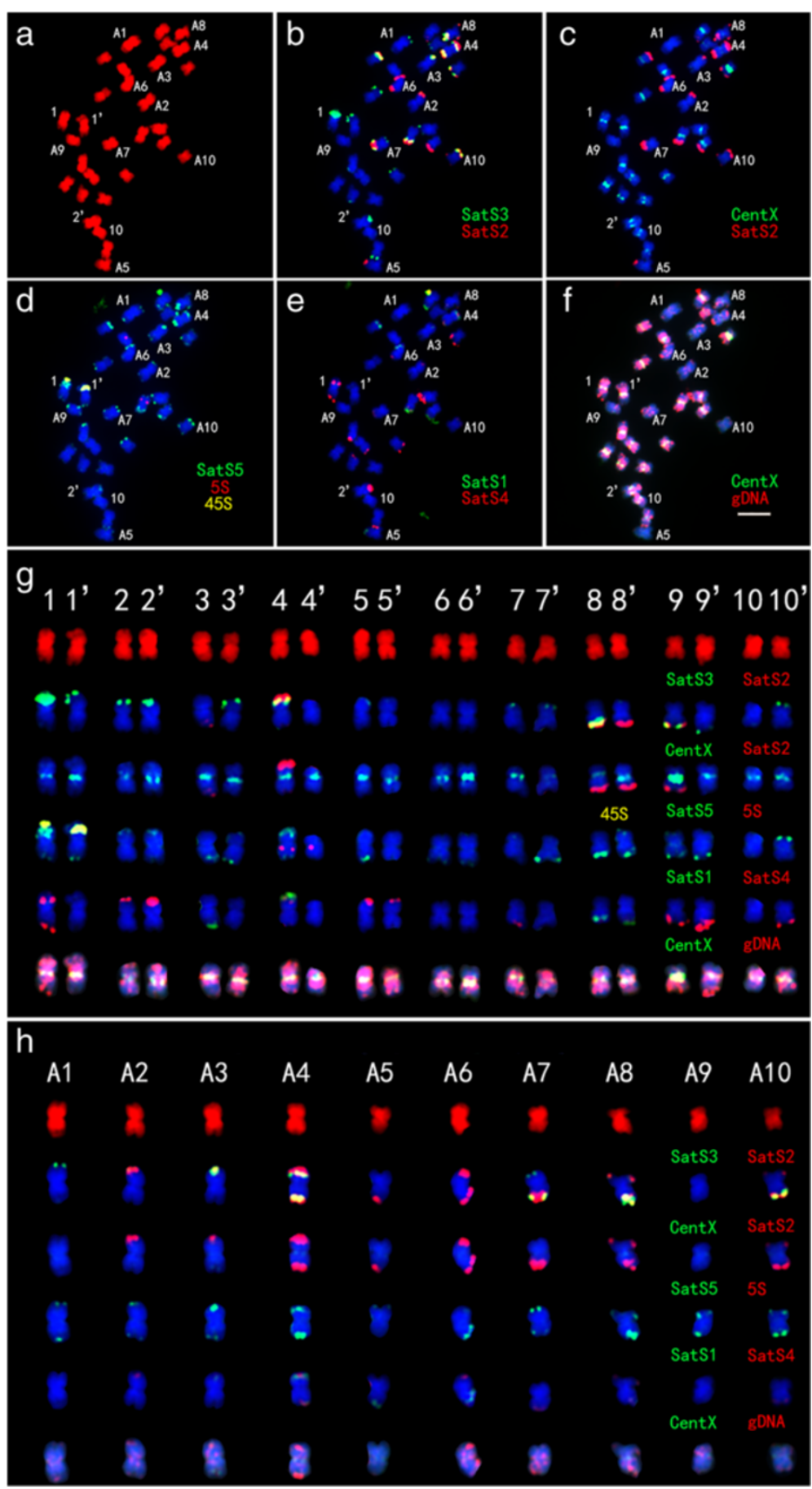

Figure 5 The karyotype for C. aquatica HG mitotic metaphase chromosomes. The karyotype for C. aquatica HG mitotic metaphase chromosomes. (a) The mitotic metaphase chromosomes (numbered from 1 to 10 ) were counterstained with DAPI and pseudocolored in red; (b) FISH with the probe cocktail of SatS3 (green) and SatS2 (red); (c) A merged imagine with CentX in green and SatS2 in red; (d) The same spread was reprobed with the probe cocktail of SatS5 (green), $5 \mathrm{~S}$ (red) and 45S (yellow); (e) The same spread was reprobed with the probe cocktails of SatS1 (green) and SatS2 (red); (f) The same spread was reprobed with the probe cocktail of CentX (green) and C. lacryma-jobi gDNA (red); (g) The twenty chromosomes closely homologous to C. lacryma-jobi were separated from figure (a-g) and listed in numerical order; (h) Ten chromosomes distant to C. lacryma-jobi were separated from figure (a-g) and listed in numerical order, bar $=5 \mu \mathrm{m}$. 

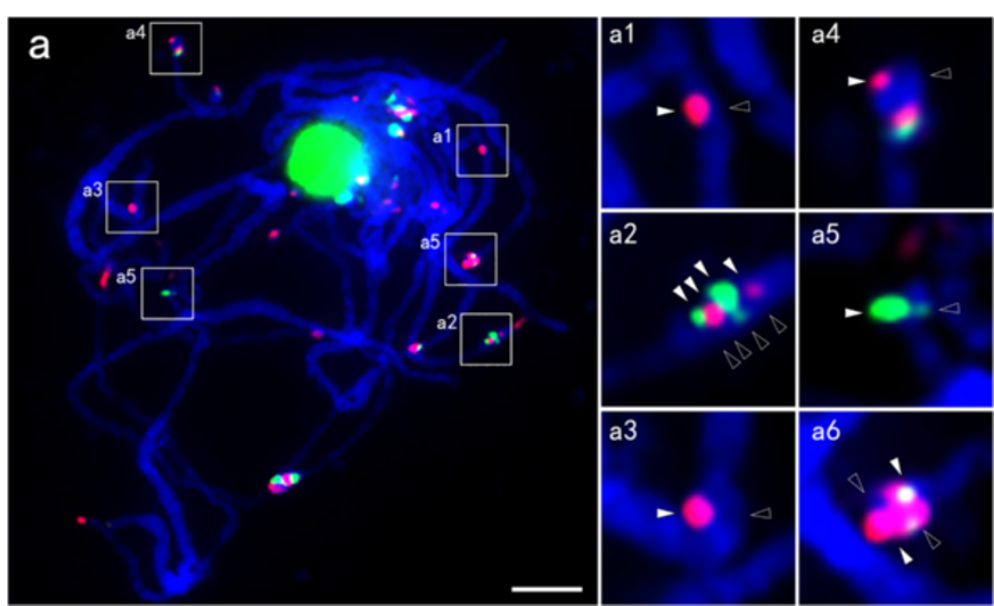

Figure 6 FISH analysis of asymmetric satellite distribution on pachytene chromosomes of C. aquatica HG. FISH analysis of asymmetric satellite distribution on pachytene chromosomes of $\mathrm{C}$. aquatica HG. The arrowhead pointed FISH signals, and the hollow arrowhead pointed the absence of the signals from the same type of probes or the obvious weaker signals. (a) Satellite staining on a pachytene chromosome of $\mathrm{C}$. aquatica HG with SatS3 (green) and SatS5 (red) probes. (a1) The enlarged image showing an unichromosomal SatS5 signal (red); (a2) The enlarged image showing two SatS5 signals (red) and two SatS3 signals (green), with one exceptionally weak SatS3 signal (green, hollow arrowhead) on a homologous chromosome; (a3) The enlarged image showing a SatS5 (red) signal on one of the synapsed chromosomes; (a4) The enlarged image showing an unichromosomal SatS5 signal (red, arrow head); (a5) The enlarged image showing a strong SatS3 signal (green, arrowhead) on one homologous chromosome and a faint one (hollow arrowhead) on the other partner; (a6) The enlarged image showing imbalanced SatS5 (red) and SatS3 (green) signals on a set of paired chromosomes, bar $=5 \mu \mathrm{m}$.

Sorghum. C. lacryma-jobi and Zea shared 43 clusters, however, these clusters only made up $0.04 \%$ of the C. lacryma-jobi genome. Thus, C. lacryma-jobi and C. aquatica HG shared more co-existing repeat families with Sorghum than with Zea. To confirm that Coix shared more repeat sequences with Sorghum than with Zea, we analyzed repeat sequences in C. lacryma-jobi, C. aquatica
HG, maize, and sorghum using the RepeatMasker program (RepBase 20130422) and found that repeat sequences in Coix were more similar to those in sorghum than to those in maize (Figure 8b). Therefore, our results corroborate the previous phylogenetic speculation, which shows that Coix might be closer to Sorghum than to Zea [14,36,37].

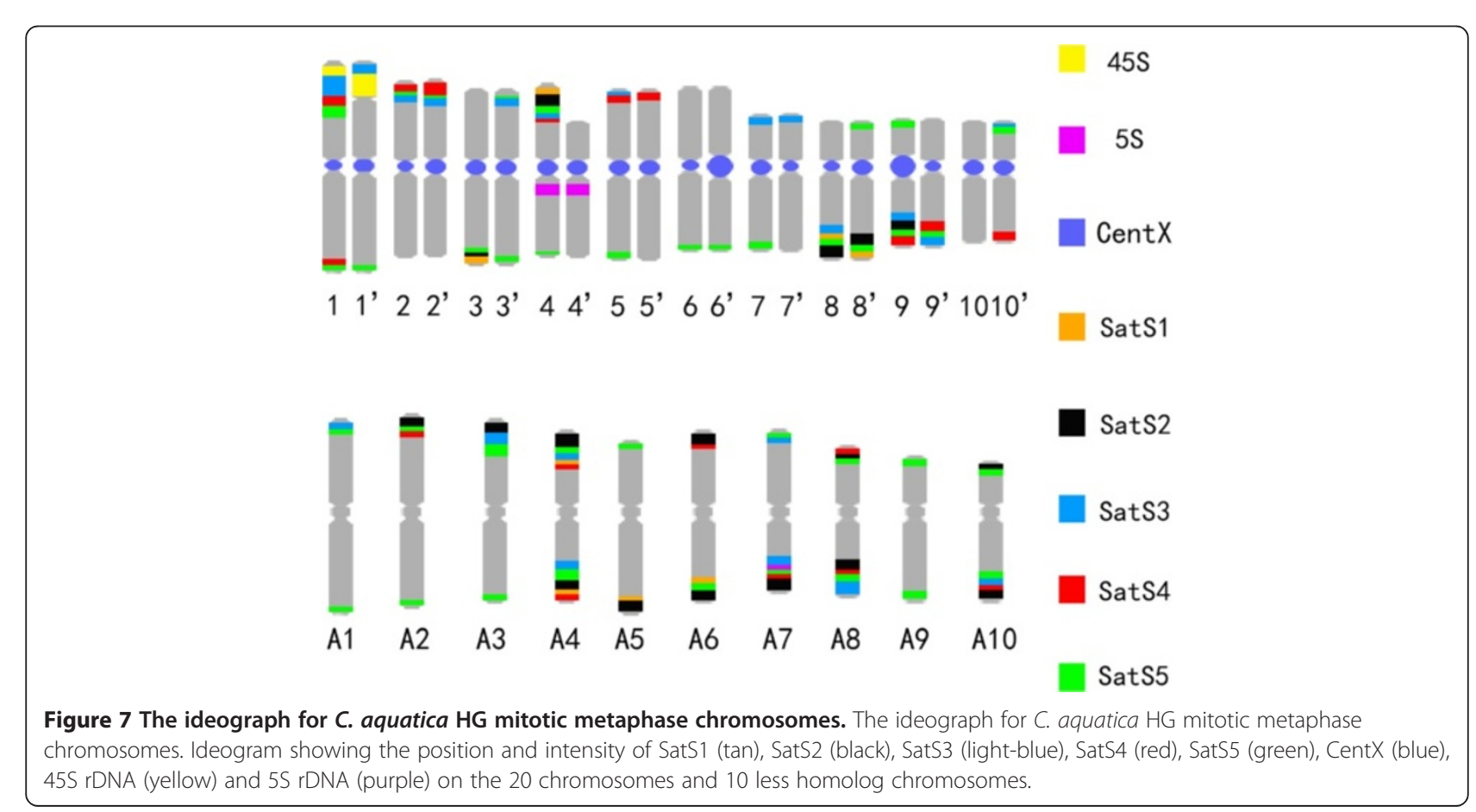


Table 4 Statistical summary of bivalents examined in meiotic metaphase I cells of C. lacryma-jobi and

\section{C. aquatica HG}

\begin{tabular}{lrlr}
\hline \multicolumn{2}{c}{ C. lacryma-jobi } & \multicolumn{2}{c}{ C. aquatica } \\
\hline 10 bivalents & 354 & 10 bivalents with 10 univalents & 31 \\
- & - & One ambiguous trivalent & 1 \\
Multivalents & 0 & Multivalents & 0 \\
Total & 354 & Total & 32 \\
\hline
\end{tabular}

\section{Discussion}

The dynamic nature of the repetitive sequences in Coix Repetitive sequences play essential roles in genome structuring and evolution, and they usually expand disproportionally to genome enlargement [38]. In the grass family, repetitive DNA accounts for $40 \%$ of $466 \mathrm{Mb}$ rice genome [39], $62 \%$ of $740 \mathrm{Mb}$ sorghum genome [19], and $82 \%$ of $2300 \mathrm{Mb}$ maize genome [18]. In general, the larger a genome size is, the larger is the portion of repeats in the genome. In this study, flow cytometry analysis showed that the genome size of C. lacryma-jobi and C. aquatica $\mathrm{HG}$, were approximately $1665 \mathrm{Mb}$ and $2335 \mathrm{Mb}$, respectively (Table 1). By using "cluster-based repeat identification" approach, we reanalyzed the repetitive DNA proportion in Zea mays and Sorghum bicolor genome as $79 \%$ and $59.4 \%$, respectively, which is consistent with the previous reports $[18,19]$. We found that Coix had a quite high percentage of repetitive DNA:
75.54\% for C. lacryma-jobi and 72.88\% for C. aquatica HG (Table 2). Notably, our results showed that C. aquatica HG with a larger genome than C. lacryma-jobi harbored less abundant repetitive DNA compared with C. lacrymajobi. One possibility might be that $C$. aquatica $\mathrm{HG}$ may arise from the hybridization of C. lacryma-jobi with another relative with less repetitive DNA, indicating a more recent origin of this species. The genome size of $C$. aquatica HG is 1.4-fold of the C. lacryma-jobi genome (Table 2). We suspected that the $C$. aquatica HG genome might be still in the process of expanding after original genome fusion. The loss of two tandem repeats (SatS1 and SatS2) and the lower percentage of the co-existing satellites in C. lacryma-jobi suggested rapid evolution of this genome (Table 3, Figures 4 and 5). The second interpretation might be that the absence of chromosome recombination and rearrangement during meiosis decelerate genome reshuffling in the sterile C. aquatica HG. Thirdly, the extent and speed of transposable element expansion may somehow vary in these two species, resulting in the compositional differences of repetitive sequences in the two species [38].

Transposable elements are a major class of repetitive DNA driving genome expansion and speciation [40]. Many families or genera have specific types of transposable elements interspersed in heterochromatic and euchromatic regions [41]. C. lacryma-jobi and C. aquatica HG consist of typical repetitive DNA in Poaceae family
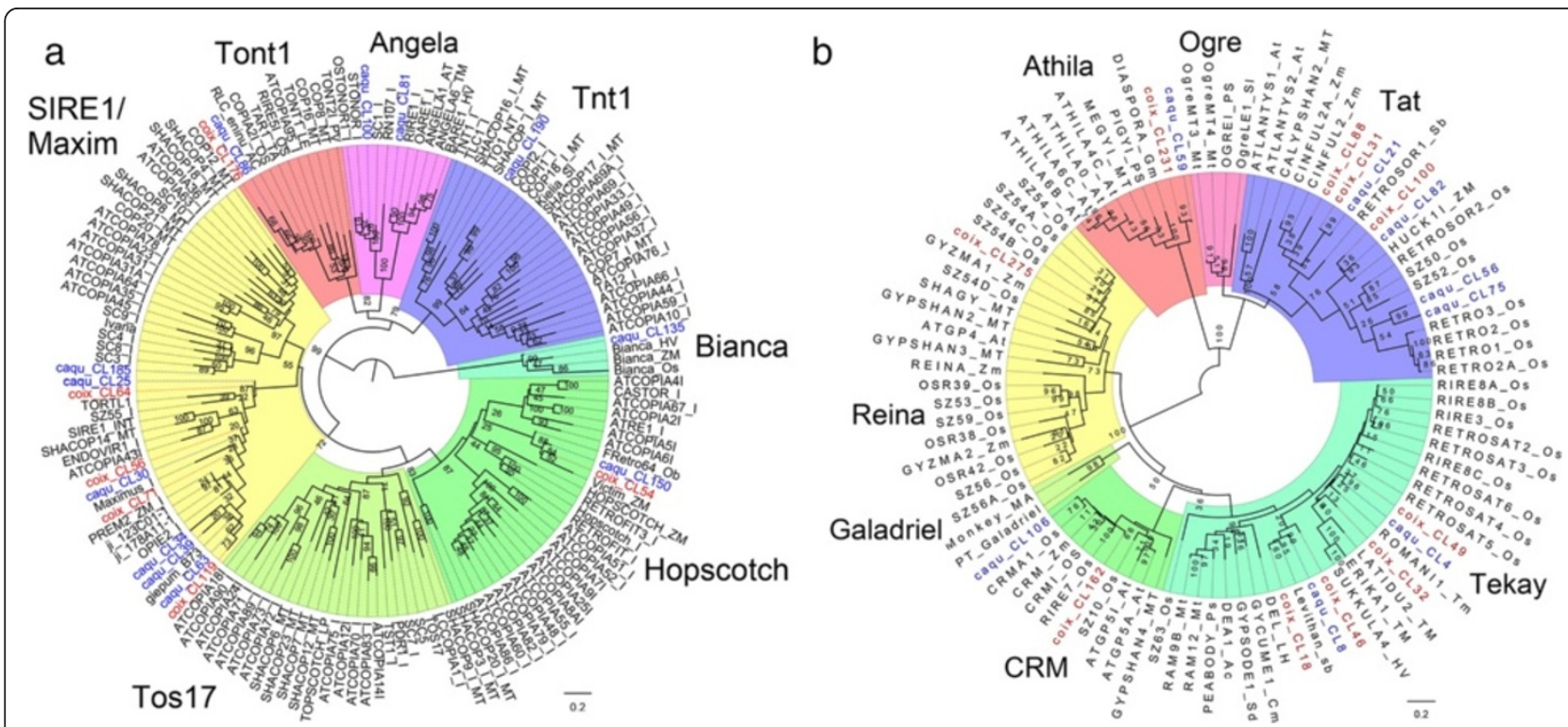

Figure 8 Phylogenetic relationships of LTR-retrotransposon elements from C. lacryma-jobi and C. aquatica HG. Phylogenetic relationships of LTR-retrotransposon elements from C. lacryma-jobi and C. aquatica HG, inferred from Neighbor-joining analysis of the reverse transcriptase encoding domain with bootstrap of 100 replicates. (a) The Ty1/Copia families from C. lacryma-jobi and C. aquatica HG. The elements from C. lacryma-jobi and C. aquatica HG were in red and blue separately; (b) The Ty3/Gypsy families from C. lacryma-jobi and C. aquatica HG. The elements from C. lacryma-jobi and C. aquatica HG were in red and blue separately. 


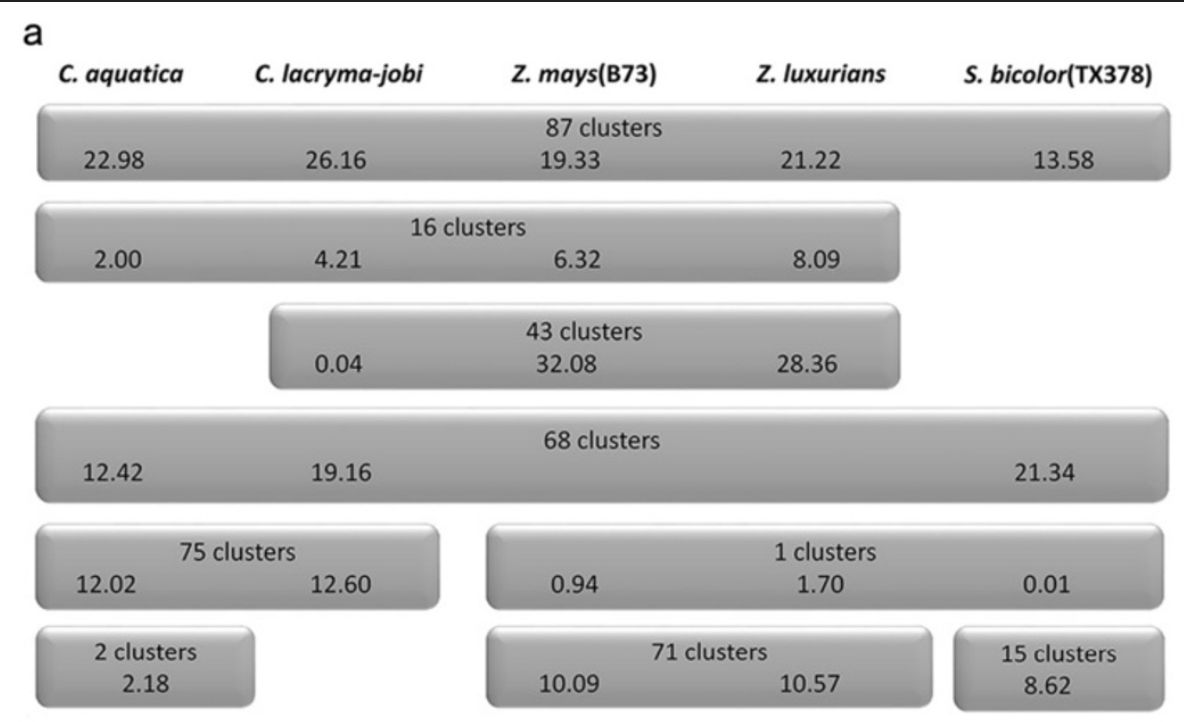

b

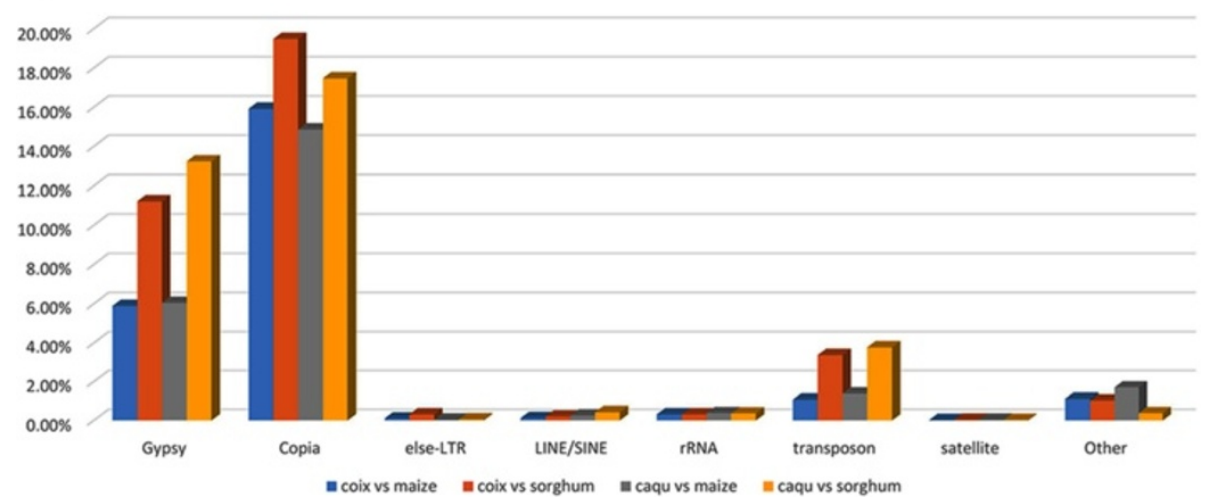

Figure 9 Comparative analysis of repeat sequences in Zea, Sorghum and Coix. Comparative analysis of repeat sequences in Zea, Sorghum, and Coix. (a) Distribution of repetitive DNA clusters in five species of Zea, Sorghum, and Coix, focusing on the 9 major distribution patterns and the corresponding numbers of clusters. (b) The RepeatMasker annotation by using Zea mays and Sorghum bicolor as the reference database.

[42]. In this study, the most abundant repetitive elements were Ty1/Copia and Ty3/Gypsy, comprising 68.68\% of the C. lacryma-jobi genome and $63.68 \%$ of the C. aquatica HG genome (Table 2). Similar to differential enrichment of Ty1/Copia and Ty3/Gypsy retroelements in Musa acuminate genome [43], we found the proportion of Ty1/ Copia was moderately higher than that of Ty3/Gypsy in C. lacryma-jobi (Table 2). However, Ty3/Gypsy retroelements were more abundant than Ty1/Copia retroelements in C. aquatica HG in our study, which is similar to Zea and Sorghum, Oryza sativa, Spirodela polyrhiza, Brachypodium distachyon, and Arabidopsis thaliana [44]. Inspection of Ty1/Copia and Ty3/Gypsy content of plant genomes in a phylogenetic context reveals no congruence in their content even in closely related species and highlights differences in success of genome occupation [45]. This obvious difference between these two species may be caused by differential amplification or removal of Ty1/
Copia and Ty3/Gypsy elements in C. lacryma-jobi and C. aquatica $\mathrm{HG}$.

Satellite repeats are also a critical class of repetitive DNA in higher eukaryotes [46]. Long stretches of satellite repeats may reside in a special chromosomal locus as a hallmark, functional unit, or a driving force for genome revolution [47]. The centromere specific satellite repeats are particularly important for centromere formation, expansion, and function [48-50]. Consistent with their functional conservation, centromeric satellite repeats are broadly conserved in length, in spite of large variation in their DNA sequences [51]. The size of individual centromeric satellite repeat is $155,165,176$, and $171 \mathrm{bp}$ in maize, rice, Brassica, and human, respectively [52-55]. Very interestingly, we found 153 and 102 bp centromeric satellite repeats in Coix. The 153 bp satellite repeat is within the normal range of the length of centromeric satellite repeats, while the 102 bp repeat is the shortest 
centromeric satellite repeat reported in Poaceae thus far. Similarly, different satellite repeats are also detected in potato [56]. In-depth comparative sequence analysis of five homologous centromeres in two Solanum species indicates that centromeric satellite repeats may undergo boom-bust cycles before a favorable repeat is fixed in a population [57]. This may explain why the $153 \mathrm{bp}$ and 102 bp repeats co-exist in the Coix centromere. Megabasesized centromeric satellite repeats in large genomes may trace back to LTR retrotransposon [58,59], although neocentromeres may function properly without satellite repeats during early stages of centromere evolution [60]. Given that $C$. aquatica HG is sterile and spreads by vegetative propagation, we believe that the evolution of its centromere may be reproductively suppressed. Therefore, the 102 bp variant may represent an evolutionary intermediate of centromeric repeat in Coix.

\section{Ploidy level of C. lacryma-jobi and C. aquatica HG}

C. lacryma-jobi $(2 \mathrm{n}=20)$ and C. aquatica HG $(2 \mathrm{n}=30)$ are generally known as tetraploid and hexaploid species, respectively, because the diploid species within the Coix genus has been reported to contain 10 chromosomes in its somatic cells [31].The genus Sorghum has 25 species with $10,20,30$, or 40 chromosomes, and these species are classified as diploid plants [61]. Ploidy level of C. lacryma-jobi and $C$. aquatica HG remains inconclusive. Karyotyping analysis has been shown to be able to provide direct evidence for homologous chromosome identification in polyploid species by visualizing characteristic chromosomal structures [62]. Taking advantage of a set of powerful satellite repeat markers, we found clear SatS3, SatS4 and SatS5 signal pairs on metaphase chromosomes in the C. lacryma-jobi karyotype, which represent the typical feature of diploid karyotypes. Furthermore, we found that 20 chromosomes displayed 10 perfect pairs in pachytene, which was consistent with the 10 bivalents during the meiosis I. Therefore, we speculated that C. lacryma-jobi might behave as a diploid species. However, our observation could not rule out the possibility that C. lacryma-jobi might be an ancient tetraploid species. During the evolution of many ancient tetraploid species, diploidization often occurs to restructure the genome $[63,64]$. Thus, we suspected that the C. lacryma-jobi genome, as a tetraploid during its early evolution, might go through subsequent diploidization, similar to what has happened to maize.

In $C$. aquatica HG, GISH with C. lacryma-jobi genomic DNA has been shown to identify 20 homologous chromosomes [7]. In this study, the 20 chromosomes also had bright centromeric satellite (CentX) signals (Figure 4). Additionally, some chromosome pairs, such as chromosome 1 and 1', and chromosomes 4 and 4', showed asymmetric repeat signals of SatS1, SatS2, SatS3, SatS4 and SatS5 (Figure 4), suggesting that the 20 paired chromosomes may be derived from a recent hybridization of two dissimilar parents and that repeat sequence homogenization may be still an ongoing process [65]. We also found that the other 10 chromosomes have extremely faint signals of centromeric satellite repeats (Figure $5 \mathrm{~h}$ ) compared with the 20 paired chromosomes, and the signal patterns of other satellites on these 10 chromosomes were also different from those on the 20 paired chromosomes in C. aquatica $\mathrm{HG}$ or in the C. lacryma-jobi chromosomes (Figures 4,5,6,7). We found that during meiosis I, 30 chromosomes of $C$. aquatica HG formed ten bivalents and ten univalents (Table 4). In addition, pollen of C. aquatica HG was almost completely sterile (Additional file 1: Table S3). All these data suggested that the $C$. aquatica HG could be a recently formed hybrid between an octoploid cytotype $(2 \mathrm{n}=40)$ related to C. lacryma-jobi and a tetraploid cytotype $(2 \mathrm{n}=20)$ of a more distantly related Coix species (Additional file 1: Figure S5). Another possibility is that the hybrid plant might be formed by a diploid $(2 n=20)$ and a different diploid $(2 \mathrm{n}=20)$ parent, consequently resulting in a numerically unreduced gamete, hence mimicking a partial autopolyploidization of the second nonhomologous genome.

\section{The phylogenetic relationship of Zea, Sorghum and Coix was corroborated by comparative repeat sequence analysis}

Zea, Sorghum, and Coix are close relatives in Poaceae family, although they independently originated in Central America, Africa, and Southeast Asia, respectively [1]. Previous studies using chloroplast sequences indicate that Coix is evolutionarily closer to Sorghum than to Zea $[14,36]$, which has been further confirmed by comparative analysis of the $b z$ orthologous region [37]. This argument, however, is still questionable due to the absence of evidence from nuclear genome. In our study, we compared all repeat elements of five species from Zea, Sorghum, and Coix. We found that the co-existing repeat families in Zea and Coix comprised only 16 clusters. However, the Sorghum and Coix shared 68 clusters. Zea and Sorghum only had 1 cluster in common (Figure 9a). These lines of evidence from nuclear DNA sequences also support a close evolutionary relationship between Coix and Sorghum and a relative distant evolutionary relationship between Zea and Sorghum.

We also identified the most abundant families of LTRretrotransposon of Zea and Sorghum in the Coix genome, indicating that Coix, Zea and Sorghum may be closely related to one another. It has been shown that $J i$ and Opie are the most abundant Ty1/Copia-type retroelements in Zea mays, and their copies decrease in Sorghum bicolor and Coix [35]. Huck is an abundant Ty3/Gypsy type retroelement in Zea mays [66], but just a moderately repetitive element in Sorghum bicolor and Coix [35]. Additionally, 
Leviathan is moderately abundant in Coix and Sorghum bicolor genomes [35]. These results, together with those from repeat sequence comparison, suggest that the dynamics of the genome-wide repeat elements in Coix, Zea and Sorghum, to certain extent, might facilitate the clarification of the evolution relationship of these close species.

\section{Conclusions}

In this study, we used low-coverage sequencing on Coix lacryma-jobi $\mathrm{L} .(2 \mathrm{n}=20)$ and Coix aquatica Roxb. HG $(2 n=30)$ to analyze repeat elements. We revealed the genome structure of these two species and large proportional difference of the same repetitive element between two genomes, indicating evolutionary divergence of these two species. The fine karyotyping analysis using satellites as markers and the chromosome pairing of meiotic metaphase I of these two species suggests that C. lacryma-jobi is likely a diploidized paleotetraploid species, and C. aquatica HG might be an early generation hybrid between Coix lacryma-jobi L. and a distantly related species. In addition, the genome-wide repeat sequence comparative analysis of Zea Mays, Zea luxurians, Sorghum bicolor, and Coix indicated that Coix seem to be closer to Sorghum than to Zea, which is consistent to previous report. Our results shed new light on the evolution and species differentiation in the Poaceae family.

\section{Methods}

\section{Plant materials}

C. lacryma-jobi cultivar BJ and C. aquatica HG were used in this study. The chromosome number and genomic features of C. lacryma-jobi and C. aquatica HG were listed in Table $1 . \mathrm{BJ}$ is the most important cultivar in north China, it is dry habitat, it's leaf blades and sheaths are broad and glabrous, it's false fruits is soft and pyriform. This phenotype is consistent to C. lacryma-jobi [15]. The $2 \mathrm{n}=30$ C. aquatica $\mathrm{HG}$ is the only C. aquatica in China, it was found in northwest of China [16]. It is perennial, aquatic, stoloniferous and rooting from the stolon node, but when grown under field conditions it becomes erect; It's leaf blades and sheaths are narrow and glabrous, the false fruits is hard and pyriform. This phenotype is consistent to C. aquatica [15].

\section{Genome size estimation}

The $1 C$ value of C. lacryma-jobi and C. aquatica HG was measured by flow cytometry with propidium iodide (PI) to stain DNA and Zea mays inbred line B73 as the reference plant. Fresh leaves were quickly cut with a sharp knife and treated with the lysis buffer $(0.18 \%$ Tris, $0.74 \% \mathrm{Na}_{2}$ EDTA, $0.01 \%$ spermine, $5.8 \% \mathrm{KCl}, 1.1 \%$ $\beta$-mercaptoethanol and $0.1 \%$ Triton X-100) for 10 minutes. The suspension was filtered with $30 \mu \mathrm{m}$ nylon mesh, centrifuged at $800 \mathrm{rpm}$ for $5 \mathrm{~min}$. The supernatant was discarded and the pellet was then resuspended in PI (Propidium Iodide, $50 \mu \mathrm{g} \cdot \mathrm{mL}^{-1}$ ). A flow cytometer (BD, FACSCalibur, USA) was used for the fluorescence measurement, with 10,000 particles measured per run and three runs performed per plant preparation. The genome size was calculated according to the formula: Genome size $_{\text {Object }}=$ (mean G1 nuclei fluorescence intensity Object $/$ mean G1 nuclei fluorescence intensitystandard ) Genome size $_{\text {Standard. }}$. The peak coefficient of variation percentages were all $<5.5 \%$.

\section{DNA extraction and Solexa sequencing}

DNA isolation was followed a standard cetyl trimethyl ammonium bromide (CTAB) extraction protocol [67]. DNA was diluted to a final concentration of $200-300 \mathrm{ng} / \mu \mathrm{l}$ for Illumina sequencing. One hundred bp paired-end reads were obtained from HiSeq2000 platform (BerryGenomics).

The sequences for Zea mays inbred line B73 SRR088692, Zea luxurians accession PI441933 SRR088692, and Sorghum bicolor inbred line Tx378 SRR561245 were downloaded from DNAnexus (http://sra.dnanexus.com/).

\section{Data access}

The genome sequencing data files associated with this study have been submitted to NCBI SRA with accession number SRP049558.

\section{Data analysis}

Sequencing data were preprocessed to remove low-quality reads, and then the unpaired reads were discarded. Repeat sequence assembly was performed using a graph-based clustering approach as described by Novak et al. [26] with the cluster size threshold for detailed analysis as $0.005 \%$. Repeat type identification was done by sequence-similarity searches of assembled contigs against Repbase repeatmaskerlibrary using RepeatMasker [68], and by detection of conserved protein domains using RPS-Blast (Reversed Position Specific-Blast) [69]. Satellite monomer within contig sequences were identified using Tandem Repeats Finder [70]. Contigs corresponding to putative mitochondrial and plastid sequences were identified by NCBI blastn and the clusters were eliminated. The genome proportion of each cluster was calculated as the percentage of reads.

To determine the distribution of different repeats between C. lacryma-jobi and C. aquatica HG, a combined dataset comprising $68,187,510$ reads and labeled reads with sample codes were built and the graph-based clustering analysis was performed as previous mentioned. To further analyze the pattern of repeats evolution between subtribe Maydeae and Sorghum, Sorghum bicolor inbred line BTX623, Zea mays inbred line B73 and Zea luxurians paired-end data were added to build another combined dataset comprising 15,000,000 reads with sample codes. Both datasets were analyzed as described above. 
The whole raw data of C. lacryma-jobi and C. aquatica HG was masked with RepeatMasker [68] using maize and sorghum as reference genomes. The threshold of identity $>70 \%$ and coverage $>50 \%$ were used to screen the output. Then the total read number for each annotation was calculated. By comparing the whole and individual repeat family between these two annotations, the relationship of these genera was analyzed.

\section{ChIP, ChIP-seq, and quantitative ChIP-PCR}

Nuclei isolation was performed according to the published protocol [71]. ChIP was performed following standard protocols [72]. Both ChIPed DNA and mock DNA were used for Solexa sequencing (BerryGenomics). ChIP-qPCR was performed to confirm relative enrichment of specific sequences within anti-OsCENH3 precipitated DNA relative to the DNA sample prepared from pre-blood immunoprecipitation $45 \mathrm{~S}$ rDNA and SatS3, localized at chromosome ends, were used as negative controls to normalize enrichment of each positive amplicon. Quantitative PCR data were analyzed as described previously [73].

\section{Repeat Identification from the ChIP-seq dataset}

To identify repeats associated with CenH3, a set of 2.5 M 100 bp paired-end ChIP-seq reads were mapped to the genomic Graph-based clusters using PatMan program [74], allowing for maximum of three mismatches including two gaps. Based on its best similarity detected among the genomic sequencing reads, each ChIP-seq read was mapped to a maximum of one cluster.

\section{FISH, GISH, and immunostaining}

Mitotic and meiotic chromosome preparation and $\mathrm{FISH}$ were performed following published protocols [75] with a minor modification. Root tips of C. lacryma-jobi and $C$. aquatica HG were harvested and immediately exposed to nitrous oxide at 2 atm for 2 hours, then fixed in a 3:1 solution of ethanol: glacial acetic acid. DNA probes for each satellite repeat and rDNA were amplified by PCR using C. lacryma-jobi and C. aquatica HG genomic DNA. Cloning of satellite repeats and rDNA were conducted using primers designed from extracted repeat clusters. The plasmids were labeled with either biotin-16-UTP, digoxigenin-11-dUTP (Roche Diagnostics), or Diethylaminocoumarin-5-dUTP (PerkinElmer) by standard nick translation. An oligonucleotide of (TTTAGGG) 3 was labeled at the 5 '-end with digoxigenin as a FISH probe to detect telomere repeat location. To determine localization of the CentX variant on C. aquatica HG chromosomes, FISH was performed with oligonucleotide AATTCAACTGCGAG TTTTTTCGTGCATCGGTGGGAAAAACGGCCCTCCC TCACGAGTTTT from the 102 bp CentX variant labeled at 5'-end with biotin and AAAACTCATGTTTGGT GGGTTTTTGGCACTTTCATTTCCG from the rest of the 51 bp labeled at 5'-end with digoxigenin. Chromosomes were counterstained with 4, 6-diamidino-2-phenylindole (DAPI) in Vectashield antifade solution (Vector Laboratories).

Images were captured digitally using a Sensys CCD camera (QIMAGING, RETIGA-SRV, FAST 1394) attached to an Olympus BX61 epifluorescence microscope (Tokyo, Japan). Image-Pro plus 6.5 software (Media Cybernetics) was used to measure FISH signals and chromosome parameters. Images were adjusted with Adobe Photoshop 7.0. For karyotype construction, chromosomes in five metaphase cells in each species were measured. Sequential FISH was conducted following published protocol [76]. After first round of FISH and image capture, the slides were washed through a set of PBS (phosphate buffer saline) buffer and ethanol series before hybridized by the second set of probes.

Immunostaining was performed following published protocols [7]. Root tips harvested from plants were fixed in $4 \%(\mathrm{w} / \mathrm{v})$ paraformaldehyde for $15 \mathrm{~min}$ at room temperature. The root tips were squashed on glass slides. The slides were incubated in a humid chamber at $37^{\circ} \mathrm{C}$ for overnight with primary antibodies against OsCENH3 diluted 1:500 in TNB buffer $(0.1 \mathrm{M}$ Tris- $\mathrm{HCl}, \mathrm{pH} 7.5$, $0.15 \mathrm{M} \mathrm{NaCl}$, and $0.5 \%$ blocking reagent). The slides were incubated with Cy3-conjugated goat anti-rabbit antibody $(1: 1000)$ at $37^{\circ} \mathrm{C}$ for $1 \mathrm{~h}$.

\section{Pollen viability}

To examine pollen viability, fresh pre-flowering panicles from $C$. aquatica HG were collected and stained with $1 \%$ iodine-potassium iodide and $1 \%$ acetocarmine solutions. More than 400 pollen grains were examined under the Olympus CX41 microscope. Stained pollen grains with a normal size were considered fertile. Small pollen grains with faint staining and empty pollen grains were considered sterile [77].

\section{LTR retrotransposon family classification}

Phylogenetic trees including representative Ty1/Copia and Ty3/Gypsy clades were reconstructed with reference sequences from the previously reported matrices [33]. For each LTR retrotransposon family, one contig covering the RT (reverse transcriptase) domain was used to represent the entire family for phylogenetic analysis. The representative contigs were selected as the most conserved considering their similarity scores with known elements obtained using RPS-BLAST and three alignment profiles: pfam07727, pfam00078 and cd01650. The amino acid sequence within the RT domain was extracted from alignment results of RT boundaries using a custom Python script. Phylogenetic trees were constructed using the Neighbor-joining method and bootstrapped with 100 replicates using the MEGA5. 


\section{Additional files}

\section{Additional file 1: Includes Table S1-S3 and the figure legends for} Figure S1-S5.

Additional file 2: List of the annotation and genome proportion of the cluster of the comparative analysis.

\section{Abbreviations}

FISH: Fluorescent in situ hybridization; GISH: Genomic in situ hybridization; Gb: Giga base pairs; Mb: Mega base pairs; bp: Base pair; RT: Reverse transcriptase; ChIP: Chromatin immunoprecipitation.

\section{Competing interests}

The authors declare that they have no competing interests.

\section{Authors' contributions}

ZC carried out molecular, cytogenetic and bioinformatic studies and drafted the manuscript. HL performed the Quantitative ChIP-PCR, participated in the cytogenetic studies and provided helpful discussions. QH participated in the ChIP assay and provided helpful discussions. MP helped to maintain the experiment materials. JC prepared the sequencing library for ChIP-DNA. $\mathrm{XL}$ and $J \mathrm{~L}$ helped to draft the manuscript and provided critical comments. WJ designed the experiment and revised the manuscript. All authors read and approved the final manuscript.

\section{Acknowledgements}

We thank Professor Jiming Jiang in the Department of Horticulture of University of Wisconsin for his critical read of the manuscript. We also thank Dr. Yingping Jiao for her help on bioinformatics analysis. This work was supported by grants from the Ministry of Science and Technology (2012AA10A305 and 2011CB944600) and the National Natural Science Foundation of China (31171563).

\section{Author details}

${ }^{1}$ National Maize Improvement Center of China, Beijing Key Laboratory of Crop Genetic Improvement, Coordinated Research Center for Crop Biology, China Agricultural University, Beijing 100193, China. ${ }^{2}$ Zhejiang Academy of Agricultural Science, Hangzhou 310021, China. ${ }^{3}$ State Key Laboratory of Agro-biotechnology and National Maize Improvement Center, Department of Plant Genetics and Breeding, China Agricultural University, Beijing 100193, China. ${ }^{4}$ Department of Plant Nutrition, China Agricultural University, Beijing 100193, China.

Received: 11 June 2014 Accepted: 19 November 2014 Published: 25 November 2014

\section{References}

1. Arora R: Job's-tears (Coix lacryma-jobi) - a minor food and fodder crop of northeastern India. (Econ Bot 1977, 31(3):358-366.

2. Ottoboni LM, Leite A, Targon MLN, Crozier A, Arruda P: Characterization of the storage protein in seed of Coix lacryma-jobi var. Adlay. J Agric Food Chem 1990, 38(3):631-635.

3. Woo J-H, Dapeng L, Orita H, Coulter J, Tully E, Kwon TK, Xu S, Gabrielson E: Coix seed extract, A commonly used treatment for cancer in china, inhibits NFKB and protein kinase $\mathrm{C}$ signaling. Cancer biology \& therapy 2007, 6(12):2005-2011.

4. Kim SO, Yun SJ, Jung B, Lee EH, Hahm DH, Shim I, Lee HJ: Hypolipidemic effects of crude extract of adlay seed (Coix lachrymajobi var. mayuen) in obesity rat fed high fat diet: relations of TNF-alpha and leptin mRNA expressions and serum lipid levels. Life Sci 2004, 75(11):1391-1404.

5. Chung CP, Hsia SM, Lee MY, Chen HJ, Cheng F, Chan LC, Kuo YH, Lin YL, Chiang W: Gastroprotective activities of adlay (Coix lachryma-jobi L. var. ma-yuen Stapf) on the growth of the stomach cancer AGS cell line and indomethacin-induced gastric ulcers. J Agric Food Chem 2011, 59(11):6025-6033.

6. Lu Y, Zhang B, Jia Z, Wu W, Lu Z: Hepatocellular carcinoma HepG2 cell apoptosis and caspase- 8 and $\mathrm{Bcl}-2$ expression induced by injectable seed extract of Coix lacryma-jobi. Hepatobiliary Pancreat Dis Int 2011, 10(3):303-307.
7. Han Y, Wang G, Liu Z, Liu J, Yue W, Song R, Zhang X, Jin W: Divergence in centromere structure distinguishes related genomes in Coix lacryma-jobi and its wild relative. Chromosoma 2010, 119(1):89-98.

8. Han Y, Li D, Li Y, Xue Y, Hu Z, Song Y: Cytogenetic identification of a new hexaploid Coix aquatica Cyto-type. Acta Bot Sin 2004, 46(6):724-729.

9. Venkateswarlu J, Rao PN: Apomictic maternal diploids in tetraploid Job's tears. Theor Appl Genet 1975, 45(6):274-276.

10. Qin F, Li J, Li X, Corke H: AFLP and RFLP linkage map in Coix. Genet Resour Crop Ev 2005, 52(2):209-214.

11. Meng X, Huang B, Zhou L, He Y, Chen Q, Yuan Y, Xu Z, Song R: Construction of a Coix BAC library and isolation of the $22 \mathrm{kDa}$ a-coixin gene cluster. Genome 2010, 53(9):667-674.

12. Ottoboni LM, Leite A, Yunes JA, Targon ML, de Souza Filho GA, Arruda P: Sequence analysis of $22 \mathrm{kDa}$-like alpha-coixin genes and their comparison with homologous zein and kafirin genes reveals highly conserved protein structure and regulatory elements. Plant Mol Biol 1993, 21(5):765-778.

13. Song R, Messing J: Contiguous genomic DNA sequence comprising the 19-kD zein gene family from maize. Plant Physiol 2002, 130(4):1626-1635

14. Kellogg EA, Watson L: Phylogenetic studies of a large data set. I. Bambusoideae, Andropogonodae, and Pooideae (Gramineae). Bot Rev 1993, 59(4):273-343.

15. Rao PN, Nirmala A: Chromosomal basis of evolution in the genus Coix $\mathrm{L}$. (Maydeae): a critical appraisal. Nucleus 2011, 53(1-2):13-24.

16. Lu P, Zuo Z: Detection and identification of Coix aquatica species in Guangxi. J Guangxi Agric Sci 1996, 1:18-20.

17. Kumar A, Bennetzen JL: Plant retrotransposons. Annu Rev Genet 1999, 33 (1):479-532.

18. Schnable PS, Ware D, Fulton RS, Stein JC, Wei F, Pasternak S, Liang C, Zhang J, Fulton L, Graves TA, Minx P, Reily AD, Courtney L, Kruchowski SS, Tomlinson C, Strong C, Delehaunty K, Fronick C, Courtney B, Rock SM, Belter E, Du F, Kim K, Abbott RM, Cotton M, Levy A, Marchetto P, Ochoa K, Jackson SM, Gillam B, et al: The B73 maize genome: complexity, diversity, and dynamics. Science 2009, 326(5956):1112-1115.

19. Paterson AH, Bowers JE, Bruggmann R, Dubchak I, Grimwood J, Gundlach H, Haberer G, Hellsten U, Mitros T, Poliakov A, Schmutz J, Spannagl M, Tang H, Wang X, Wicker T, Bharti AK, Chapman J, Feltus FA, Gowik U, Grigoriev IV, Lyons E, Maher CA, Martis M, Narechania A, Otillar RP, Penning BW, Salamov AA, Wang Y, Zhang L, Carpita NC, et al: The Sorghum bicolor genome and the diversification of grasses. Nature 2009, 457(7229):551-556.

20. Wolfgruber TK, Sharma A, Schneider KL, Albert PS, Koo D-H, Shi J, Gao Z, Han F, Lee $H, X u$ R: Maize centromere structure and evolution: sequence analysis of centromeres 2 and 5 reveals dynamic loci shaped primarily by retrotransposons. PLoS Genet 2009, 5(11):e1000743.

21. Ganal M, Riede I, Hemleben V: Organization and sequence analysis of two related satellite DNAs in cucumber (Cucumis sativus L.). J Mol Evol 1986, 23(1):23-30.

22. Sharma S, Raina SN: Organization and evolution of highly repeated satellite DNA sequences in plant chromosomes. Cytogenet Genome Res 2005, 109(1-3):15-26.

23. Albert PS, Gao Z, Danilova TV, Birchler JA: Diversity of chromosomal karyotypes in maize and its relatives. Cytogenet Genome Res 2010, 129(1-3):6-16

24. Kato A, Lamb JC, Birchler JA: Chromosome painting using repetitive DNA sequences as probes for somatic chromosome identification in maize. Proc Natl Acad Sci USA 2004, 101(37):13554-13559.

25. Bennett MD, Leitch IJ: Nuclear DNA Amounts in Angiosperms. Ann Bot 1995, 76(2):113-176.

26. Novak P, Neumann P, Macas J: Graph-based clustering and characterization of repetitive sequences in next-generation sequencing data. BMC Bioinformatics 2010, 11:378.

27. Wicker T, Sabot F, Hua-Van A, Bennetzen JL, Capy P, Chalhoub B, Flavell A, Leroy P, Morgante M, Panaud O: A unified classification system for eukaryotic transposable elements. Nat Rev Genet 2007, 8(12):973-982.

28. Renny-Byfield S, Kovarik A, Kelly L, Macas J, Novak P, Chase MW, Nichols RA, Pancholi MR, Grandbastien MA, Leitch AR: Diploidization and genome size change in allopolyploids is associated with differential dynamics of low- and high-copy sequences. Plant J 2013, 74(5):829-839.

29. Renny-Byfield S, Chester M, Kovarik A, Le Comber SC, Grandbastien MA, Deloger M, Nichols RA, Macas J, Novak P, Chase MW, Leitch AR: Next generation sequencing reveals genome downsizing in allotetraploid 
Nicotiana tabacum, predominantly through the elimination of paternally derived repetitive DNAs. Mol Biol Evol 2011, 28(10):2843-2854.

30. Ozkan H, Levy AA, Feldman M: Allopolyploidy-induced rapid genome evolution in the wheat (Aegilops-Triticum) group. Plant Cell 2001, 13(8):1735-1747

31. Koul A: Heterochromatin and non-homologous chromosome associations in Coix aquatica. Chromosoma 1964, 15(3):243-245.

32. Sapre A, Deshpande D: Isolation of a series of chromosomal variants $(2 n=10$ to 21$)$ from an open-pollinated population of interspecific hybrids between Coix gigantea and Coix aquatica (Poaceae). Genetica 1987, 74(1):61-68.

33. Piednoel M, Carrete-Vega G, Renner SS: Characterization of the LTR retrotransposon repertoire of a plant clade of six diploid and one tetraploid species. Plant J 2013, 75(4):699-709.

34. Bousios A, Kourmpetis YA, Pavlidis P, Minga E, Tsaftaris A, Darzentas N: The turbulent life of Sirevirus retrotransposons and the evolution of the maize genome: more than ten thousand elements tell the story. Plant $J$ 2012, 69(3):475-488.

35. Estep MC, DeBarry JD, Bennetzen JL: The dynamics of LTR retrotransposon accumulation across 25 million years of panicoid grass evolution. Heredity (Edinb) 2013, 110(2):194-204.

36. Leseberg $\mathrm{CH}$, Duvall MR: The complete chloroplast genome of Coix lacryma-jobi and a comparative molecular evolutionary analysis of plastomes in cereals. J Mol Evol 2009, 69(4):311-318.

37. Wang Q, Dooner HK: Dynamic evolution of bz orthologous regions in the Andropogoneae and other grasses. Plant J 2012, 72(2):212-221.

38. Kidwell MG: Transposable elements and the evolution of genome size in eukaryotes. Genetica 2002, 115(1):49-63.

39. Yu J, Hu S, Wang J, Wong GK, Li S, Liu B, Deng Y, Dai L, Zhou Y, Zhang X, Cao M, Liu J, Sun J, Tang J, Chen Y, Huang X, Lin W, Ye C, Tong W, Cong L, Geng J, Han Y, Li L, Li W, Hu G, Huang X, Li W, Li J, Liu Z, Li L, et al: A draft sequence of the rice genome (Oryza sativa L. ssp. indica). Science 2002, 296(5565):79-92

40. Fedoroff N: Transposons and genome evolution in plants. Proc Natl Acad Sci U S A 2000, 97(13):7002-7007.

41. Baucom RS, Estill JC, Chaparro C, Upshaw N, Jogi A, Deragon J-M, Westerman RP, SanMiguel PJ, Bennetzen JL: Exceptional diversity, non-random distribution, and rapid evolution of retroelements in the B73 maize genome. PLoS Genet 2009, 5(11):e1000732.

42. Ouyang S, Buell CR: The TIGR Plant Repeat Databases: a collective resource for the identification of repetitive sequences in plants. Nucleic Acids Res 2004, 32(suppl 1):D360-D363.

43. Hřibová E, Neumann P, Matsumoto T, Roux N, Macas J, Doležel J: Repetitive part of the banana (Musa acuminata) genome investigated by low-depth 454 sequencing. BMC Plant Biol 2010, 10(1):204

44. Wang W, Haberer G, Gundlach H, Glasser C, Nussbaumer T, Luo MC, Lomsadze A, Borodovsky M, Kerstetter RA, Shanklin J, Byrant DW, Mockler TC, Appenroth KJ, Grimwood J, Jenkins J, Chow J, Choi C, Adam C, Cao XH Fuchs J, Schubert I, Rokhsar D, Schmutz J, Michael TP, Mayer KF, Messing J: The Spirodela polyrhiza genome reveals insights into its neotenous reduction fast growth and aquatic lifestyle. Nat Commun 2014, 5:3311.

45. Vitte C, Fustier MA, Alix K, Tenaillon MI: The bright side of transposons in crop evolution. Brief Funct Genomics 2014, 13(4):276-295

46. Shapiro JA, Sternberg R: Why repetitive DNA is essential to genome function. Biol Rev 2005, 80(2):227-250.

47. Plohl M, Luchetti A, Meštrović N, Mantovani B: Satellite DNAs between selfishness and functionality: structure, genomics and evolution of tandem repeats in centromeric (hetero) chromatin. Gene 2008, 409(1):72-82.

48. Harrington JJ, Van Bokkelen G, Mays RW, Gustashaw K, Willard HF: Formation of de novo centromeres and construction of first-generation human artificial microchromosomes. Nat Genet 1997, 15(4):345-355.

49. Jin W, Melo JR, Nagaki K, Talbert PB, Henikoff S, Dawe RK, Jiang J: Maize centromeres: organization and functional adaptation in the genetic background of oat. Plant Cell 2004, 16(3):571-581.

50. Wu J, Yamagata H, Hayashi-Tsugane M, Hijishita S, Fujisawa M, Shibata M, Ito $Y$, Nakamura M, Sakaguchi M, Yoshihara R, Kobayashi H, Ito K, Karasawa W, Yamamoto M, Saji S, Katagiri S, Kanamori H, Namiki N, Katayose Y, Matsumoto T, Sasaki T: Composition and structure of the centromeric region of rice chromosome 8. Plant Cell 2004, 16(4):967-976.

51. Henikoff S, Ahmad K, Malik HS: The centromere paradox: stable inheritance with rapidly evolving DNA. Science 2001, 293(5532):1098-1102.
52. Zhang W, Yi C, Bao W, Liu B, Cui J, Yu H, Cao X, Gu M, Liu M, Cheng Z: The transcribed 165 -bp CentO satellite is the major functional centromeric element in the wild rice species Oryza punctata. Plant Physiol 2005, 139(1):306-315

53. Cheng Z, Dong F, Langdon T, Ouyang S, Buell CR, Gu M, Blattner FR, Jiang J: Functional rice centromeres are marked by a satellite repeat and a centromere-specific retrotransposon. Plant Cell 2002, 14(8):1691-1704.

54. Lim K-B, De Jong H, Yang T-J, Park J-Y, Kwon S-J, Kim JS, Lim M-H, Kim JA, Jin M, Jin Y-M: Characterization of rDNAs and tandem repeats in the heterochromatin of Brassica rapa. Mol Cells 2005, 19(3):436-444.

55. Masumoto H, Masukata H, Muro Y, Nozaki N, Okazaki T: A human centromere antigen (CENP-B) interacts with a short specific sequence in alphoid DNA, a human centromeric satellite. J Cell Biol 1989, 109(5):1963-1973.

56. Gong Z, Wu Y, Koblizkova A, Torres GA, Wang K, lovene M, Neumann P, Zhang W, Novak P, Buell CR, Macas J, Jiang J: Repeatless and repeat-based centromeres in potato: implications for centromere evolution. Plant Cell 2012, 24(9):3559-3574.

57. Zhang H, Koblizkova A, Wang K, Gong Z, Oliveira L, Torres GA, Wu Y, Zhang W, Novak P, Buell CR, Macas J, Jiang J: Boom-Bust Turnovers of Megabase-Sized Centromeric DNA in Solanum Species: Rapid Evolution of DNA Sequences Associated with Centromeres. Plant Cell 2014, 26(4):1436-1447.

58. Tek AL, Song J, Macas J, Jiang J: Sobo, a recently amplified satellite repeat of potato, and its implications for the origin of tandemly repeated sequences. Genetics 2005, 170(3):1231-1238.

59. Sharma A, Wolfgruber TK, Presting GG: Tandem repeats derived from centromeric retrotransposons. BMC Genomics 2013, 14(1):142.

60. Zhang B, Lv Z, Pang J, Liu Y, Guo X, Fu S, Li J, Dong Q, Wu HJ, Gao Z, Wang $X J$, Han F: Formation of a functional maize centromere after loss of centromeric sequences and gain of ectopic sequences. Plant Cell 2013, 25(6):1979-1989.

61. Price HJ, Dillon SL, Hodnett G, Rooney WL, Ross L, Johnston JS: Genome evolution in the genus Sorghum (Poaceae). Ann Bot 2005, 95(1):219-227.

62. Xiong Z, Pires JC: Karyotype and identification of all homoeologous chromosomes of allopolyploid Brassica napus and its diploid progenitors. Genetics 2011, 187(1):37-49.

63. Clarkson JJ, Lim KY, Kovarik A, Chase MW, Knapp S, Leitch AR: Long-term genome diploidization in allopolyploid Nicotiana section Repandae (Solanaceae). New Phytol 2005, 168(1):241-252.

64. Mandakova T, Joly S, Krzywinski M, Mummenhoff K, Lysak MA: Fast diploidization in close mesopolyploid relatives of Arabidopsis. Plant Cell 2010, 22(7):2277-2290

65. Lim KY, Soltis DE, Soltis PS, Tate J, Matyasek R, Srubarova H, Kovarik A, Pires JC, Xiong Z, Leitch AR: Rapid chromosome evolution in recently formed polyploids in Tragopogon (Asteraceae). PLoS One 2008, 3(10):e3353.

66. Meyers BC, Tingey SV, Morgante M: Abundance, distribution, and transcriptional activity of repetitive elements in the maize genome. Genome Res 2001, 11(10):1660-1676.

67. Doyle JJ: A rapid DNA isolation procedure for small quantities of fresh leaf tissue. Phytochem bull 1987, 19:11-15.

68. Smit AF, Hubley R, Green P: RepeatMasker Open-3.0. 1996-2010. http://www.repeatmasker.org.

69. Altschul SF, Madden TL, Schäffer AA, Zhang J, Zhang Z, Miller W, Lipman DJ: Gapped BLAST and PSI-BLAST: a new generation of protein database search programs. Nucleic Acids Res 1997, 25(17):3389-3402.

70. Benson G: Tandem repeats finder: a program to analyze DNA sequences. Nucleic Acids Res 1999, 27(2):573.

71. Zhang W, Wu Y, Schnable JC, Zeng Z, Freeling M, Crawford GE, Jiang J: High-resolution mapping of open chromatin in the rice genome. Genome Res 2012, 22(1):151-162.

72. Nagaki K, Talbert PB, Zhong CX, Dawe RK, Henikoff S, Jiang J: Chromatin immunoprecipitation reveals that the 180-bp satellite repeat is the key functional DNA element of Arabidopsis thaliana centromeres. Genetics 2003, 163(3):1221-1225.

73. Wang G, He Q, Liu F, Cheng Z, Talbert PB, Jin W: Characterization of CENH3 proteins and centromere-associated DNA sequences in diploid and allotetraploid Brassica species. Chromosoma 2011, 120(4):353-365.

74. Prufer K, Stenzel U, Dannemann M, Green RE, Lachmann M, Kelso J: PatMaN: rapid alignment of short sequences to large databases. Bioinformatics 2008, 24(13):1530-1531.

75. Zhao X, Lu J, Zhang Z, Hu J, Huang S, Jin W: Comparison of the distribution of the repetitive DNA sequences in three variants of 
Cucumis sativus reveals their phylogenetic relationships. I Genet Genomics 2011, 38(1):39-45.

76. Liu C, Liu J, Li H, Zhang Z, Han Y, Huang S, Jin W: Karyotyping in melon (Cucumis melo L.) by cross-species fosmid fluorescence in situ hybridization. Cytogenet Genome Res 2010, 129(1-3):241-249.

77. Kubo T, Yoshimura A, Kurata N: Hybrid male sterility in rice is due to epistatic interactions with a pollen killer locus. Genetics 2011, 189(3):1083-1092.

doi:10.1186/1471-2164-15-1025

Cite this article as: Cai et al.: Differential genome evolution and speciation of Coix lacryma-jobi L. and Coix aquatica Roxb. hybrid guangxi revealed by repetitive sequence analysis and fine karyotyping. BMC Genomics 2014 15:1025.

\section{Submit your next manuscript to BioMed Central and take full advantage of:}

- Convenient online submission

- Thorough peer review

- No space constraints or color figure charges

- Immediate publication on acceptance

- Inclusion in PubMed, CAS, Scopus and Google Scholar

- Research which is freely available for redistribution 